\title{
A Comparison of Various Energy and Protein Concentrations in Diets on the Performance, Bone Mineral Density and Blood Characteristics of Broiler Chicks
}

\author{
Ho Sung Choe ${ }^{1}$, Jae Sang $\mathrm{Um}^{2}$ and Kyeong Seon Ryu ${ }^{3,+}$ \\ ${ }^{1}$ Department of Animal Biotechnology, Chonbuk National University, Jeonju 561-756 Korea, \\ ${ }^{2}$ Nonghyup Feed Inc., Seoul 134-763 Korea, \\ ${ }^{3}$ Department of Animal Science, Chonbuk National University, Jeonju 561-756 Korea
}

\begin{abstract}
Two experiments were conducted separately with different concentration of dietary energy and protein to evaluate the performance, blood characteristics and bone mineral density (BMD) of broiler chicks. In experimentr 1, a total of 480 heads one-day-old Ross $\times$ Ross broiler chicks were randomly divided into 6 treatments ( 5 replications; 16 birds/pen). Three concentration of ME $(3,000,3,100$ and 3,200 kcal $/ \mathrm{kg}$ ) and two of CP (pre-starter 22, 23\%, starter 20,21\% and finisher $18,19 \%)$ in a $3 \times 2$ factorial arrangement of treatments were used. In experiment 2, similar chicks and $\mathrm{CP}$ concentration was used but ME concentration was changed (pre-starter; 3,000, 3,050 and 3,100 kcal/kg, starter; 3,050, 3,100 and 3,150 $\mathrm{kcal} / \mathrm{kg}$, finisher; $3,100,3,150$ and $3,200 \mathrm{kcal} / \mathrm{kg}$ ) in the diet. In both experiments, 10 blood and tibia samples were collected per treatment and blood characteristics and BMD were analyzed. In experiment 1, weight gain and feed intake were increased by the $3,000 \mathrm{kcal} / \mathrm{kg} \mathrm{ME}$ in the diet $(P<0.05)$. Serum total protein and albumin levels were increased numerically with the level of CP in the diet. Total cholesterol and HDL content were increased numerically with the energy content in the diet. Consistently in experiment 2, weight gain was increased numerically by the energy and protein level (prestarter 3,000 $\times 23$, starter 3,050 $\times 21$ and finisher 3,100 kcal $/ \mathrm{kg} \mathrm{ME}$ and $19 \% \mathrm{CP}$ ) in the diet. Serum glucose level was increased with the energy level in the diet $(P<0.05)$. Therefore, serum total protein, albumin, triglycerides, total cholesterol and HDL contents were tended to increase with the energy increments in the diet.
\end{abstract}

(Key words: energy, protein, performance, blood composition, broiler chicks)

\section{INTRODUCTION}

During the last decades, genetic selection programs, improvement of nutrition and rearing environments may enhance growth performance of broiler chicks (Jang et al., 2009). Therefore, most of the nutrient requirements released by NRC (1994) may be slightly low for maximizing the growth yields of broiler chicks. But, dietary energy and protein contributing ingredients have played a major role of feed cost. Higher dietary level of ME and CP is costly, whereas lower level may affect growth performance. On the other hand, high dietary energy content in the diet caused deposition of excess abdominal and carcass fat and low dietary protein have decreased meat yields and increased fat deposition (Tumova and Teimouri, 2010). In the Republic of Korea, the commercial poultry industry has greatly increased and broiler chicks have reached 1.8 to $2.0 \mathrm{~kg}$ body weight at $33 \sim 35$ days (Jang et al., 2009).
To meet the domestic demands in Korea, major feed ingredients have been imported from USA, Brazil and China etc. Therefore, considering the high cost of feed, it is important to optimize energy and protein levels to maximize growth and meat yield of broiler chicks.

In commercial production, different levels of ME $(2,926$ to $3,300 \mathrm{kcal} / \mathrm{kg}$ ) and CP (16 to $23 \%$ ) are used in formulating broiler diets (Saleh et al., 2004a,b, Pesti, 2009). Furthermore, the efficiency of energy use decreases as the bird advances with age (Hidalgo et al., 2004). Several researchers (Saleh et al., 2004, Griffiths et al., 1977 and Bartov et al., 1974) mentioned that increasing concentrations of dietary ME while maintaining a constant ratio to $\mathrm{CP}$ of broiler diets improves feed conversion without increasing fat deposition. Because providing nutrients at either an excessive or marginal concentration to the bird's requirement can depress broiler growth. On the other hand, the response of broiler chicks to dietary

\footnotetext{
${ }^{\dagger}$ To whom correspondence should be addressed : seon@jbnu.ac.kr
} 
ME and CP may be different according to the stage of growth and bone development. But, most previous researchers did not evaluate the level of energy and protein in different growth stage (prestarter, starter and grower) of broiler chicks. Meanwhile, it is an important issue to optimize the dietary energy and protein level with different growth stage of modern broiler chicks. Therefore, two experiments were conducted consecutively to optimize the levels of dietary ME and $\mathrm{CP}$ on the performance, bone mineral density and blood characteristics composition of broiler chicks.

\section{MATERIALS AND METHODS}

\section{Birds and Management}

This experiment was conducted in an experimental farm, Department of Animal Science, Chonbuk National University, Korea. Chicks were bedded with rice husk and stocking density $\left(10\right.$ birds $\left./ \mathrm{m}^{2}\right)$ was maintained in each pen. Supplementary heat was provided by hot water pipes $(85 \mathrm{~cm})$ under the floor. The room temperature was maintained at $33^{\circ} \mathrm{C}$ for the first week, and then reduced by $2 \sim 3^{\circ} \mathrm{C}$ per week until it reached $22^{\circ} \mathrm{C}$ on $28 \mathrm{~d}$, which was maintained until the end of the experiment. The mean relative humidity was 60 to $65 \%$ and was kept constant within this range throughout the experiment. Chicks had ad libitum access to commercial pellet and water, and diets were formulated to meet the nutrient requirements according to NRC (1994). When the set temperature and humidity were exceeded, fan was operated automatically and circulated fresh air inside the pen. To prevent any preferences for familiar odors interfering with the treatment, each pen were cleaned daily. During the two experiments, body weight and feed intake were determined at weekly intervals. The feed conversion ratio was calculated by dividing the feed intake and weight gain. All managements of chicks and experimental procedures were conducted in accordance with the Institutional Animal Care and Use Committee at Chonbuk National University, Korea.

\section{Experiment 1}

A total of 480 day One - day-old Ross $\times$ Ross broiler chicks (39.54 $\pm 0.49 \mathrm{~g})$ were arranged in a factorial design
$(3 \times 2)$ in which three energy $(3,000,3,100$ and 3,200 kcal/ $\mathrm{kg} \mathrm{ME}$ ) and two protein levels (pre-starter 22, 23\%, starter $20,21 \%$ and finisher 18, 19\% CP). Each treatment was replicated 5 times with 16 chicks per replicate. The composition of the experimental diets is shown in Table 1.

\section{Blood Characteristics and Bone Mineral Den- sity}

Ten birds from each treatment were selected according to mean body weight and blood samples were taken by puncturing the wing vein, and the serum was collected at the end of the experiment, and stored at $-70^{\circ} \mathrm{Cuntil}$ analysis. Biochemical blood parameters, including glucose (GLU, mg/dL), total protein (TP, g/dL), albumin (ALB, g/dL), total cholesterol (TCL, mg/dL), triglycerides (TG, mg/dL), high density lipoprotein (HDL, mg/dL), aspartate aminotransferase (AST, U/I) and alanine aminotransferase (ALT, U/I) concentrations were measured on a Konelab 20 analyzer (Thermo Fisher Scientific, Vantaa, Finland) following the manufacturer's instructions. At 5 weeks of age, 10 chicks per treatment were killed by cervical dislocation and the tibia was removed from the muscle. Bone mineral density of the tibia was measured by bone densitometry (pDEXA, Norland Medical Systems Inc., White Plains, NY, USA).

\section{Experiment 2}

In experiment 1 , chicks were provided three level of $\mathrm{ME}$ $(3,000,3,100$ and $3,200 \mathrm{kcal} / \mathrm{kg})$ and two level of $\mathrm{CP}$ during the 35 days rearing period and identified that the $3,000 \mathrm{kcal} /$ $\mathrm{kg}$ did enhance chick's performance. Therefore, in experiment 2 design was the same protein in diets as the Expt 1 except the ME concentration (pre-starter : 3,000, 3,050 and 3,100 $\mathrm{kcal} / \mathrm{kg}$, starter : 3,050, 3,100 and 3,150 kcal/kg, finisher : $3,100,3,150$ and $3,200 \mathrm{kcal} / \mathrm{kg}$ ) in the diet. All other procedures were as described for experiment 1 . Blood and bone samples were collected and analyzed using the procedures as described for experiment 1 .

\section{Statistical Analysis}

In both experiments, data were analyzed as $3 \times 2$ (3 levels of ME and 2 levels of dietary protein) arrangement of treat- 
Table 1. General diet composition of experiment 1

\begin{tabular}{|c|c|c|c|c|c|c|c|c|c|c|c|c|c|c|c|c|c|c|}
\hline \multirow{2}{*}{$\begin{array}{l}\begin{array}{c}\text { Ingredients } \\
(\%)\end{array} \\
\text { Corn }\end{array}$} & \multicolumn{6}{|c|}{ Pre-starter $(0 \sim 1$ weeks $)$} & \multicolumn{6}{|c|}{ Starter $(2 \sim 3$ weeks $)$} & \multicolumn{6}{|c|}{ Finisher $(4 \sim 5$ weeks $)$} \\
\hline & 54.18 & 54.53 & 54.88 & 51.93 & 52.38 & 52.84 & 59.89 & 59.73 & 59.57 & 57.84 & 57.68 & 57.52 & 63.71 & 62.88 & 62.72 & 60.98 & 60.82 & 60.66 \\
\hline $\begin{array}{l}\text { Soybean } \\
\text { meal }\end{array}$ & 36.07 & 31.80 & 27.42 & 37.51 & 32.76 & 28.02 & 29.87 & 26.27 & 22.67 & 30.48 & 26.87 & 23.27 & 24.68 & 21.08 & 17.48 & 25.28 & 21.68 & 18.08 \\
\hline $\begin{array}{l}\text { Corn gluten } \\
\text { meal }\end{array}$ & - & 2.998 & 6.134 & - & 4.157 & 7.547 & 0.254 & 2.894 & 5.535 & 1.673 & 4.313 & 6.954 & - & 2.315 & 4.956 & 1.103 & 3.744 & 6.384 \\
\hline Wheat & 3.000 & 3.000 & 3.000 & 3.000 & 3.000 & 3.000 & 5.000 & 5.000 & 5.000 & 5.000 & 5.000 & 5.000 & 5.000 & 5.000 & 5.000 & 5.000 & 5.000 & 5.000 \\
\hline Wheat bran & - & - & - & - & - & - & - & - & - & - & - & - & 3.000 & 3.000 & 3.000 & 3.000 & 3.000 & 3.000 \\
\hline Soybean oil & 2.500 & 3.250 & 4.000 & 2.500 & 3.250 & 4.000 & 1.000 & 2.000 & 3.000 & 1.000 & 2.000 & 3.000 & 1.000 & 2.000 & 3.000 & 1.000 & 2.000 & 3.000 \\
\hline Limestone & 1.526 & 1.538 & 1.550 & 1.524 & 1.537 & 1.550 & 1.269 & 1.277 & 1.286 & 1.268 & 1.277 & 1.286 & 1.412 & 1.421 & 1.429 & 1.412 & 1.421 & 1.429 \\
\hline DCP & 1.696 & 1.757 & 1.818 & 1.700 & 1.743 & 1.786 & 1.784 & 1.818 & 1.852 & 1.773 & 1.807 & 1.841 & 1.263 & 1.297 & 1.332 & 1.252 & 1.286 & 1.321 \\
\hline Salt & 0.390 & 0.395 & 0.390 & 0.398 & 0.393 & 0.387 & 0.397 & 0.393 & 0.389 & 0.394 & 0.390 & 0.386 & 0.398 & 0.395 & 0.391 & 0.395 & 0.392 & 0.388 \\
\hline Lysine & 0.263 & 0.381 & 0.499 & 0.291 & 0.418 & 0.546 & 0.208 & 0.306 & 0.403 & 0.251 & 0.349 & 0.446 & 0.210 & 0.308 & 0.405 & 0.248 & 0.346 & 0.443 \\
\hline Methionine & 0.161 & 0.142 & 0.123 & 0.166 & 0.145 & 0.124 & 0.119 & 0.103 & 0.087 & 0.119 & 0.103 & 0.087 & 0.116 & 0.100 & 0.084 & 0.116 & 0.100 & 0.084 \\
\hline $\begin{array}{l}\text { Vitamin } \\
\text { premix }^{1}\end{array}$ & 0.100 & 0.100 & 0.100 & 0.100 & 0.100 & 0.100 & 0.100 & 0.100 & 0.100 & 0.100 & 0.100 & 0.100 & 0.100 & 0.100 & 0.100 & 0.100 & 0.100 & 0.100 \\
\hline $\begin{array}{l}\text { Mineral } \\
\text { premix }^{2}\end{array}$ & 0.100 & 0.100 & 0.100 & 0.100 & 0.100 & 0.100 & 0.100 & 0.100 & 0.100 & 0.100 & 0.100 & 0.100 & 0.100 & 0.100 & 0.100 & 0.100 & 0.100 & 0.100 \\
\hline Total & & & & & & & ----- & 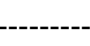 & --100. & .000 & 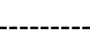 & 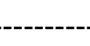 & & & & & & \\
\hline
\end{tabular}

Chemical composition

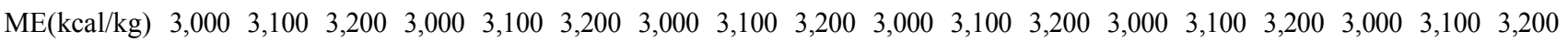

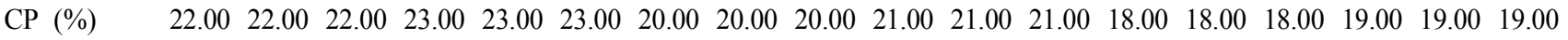

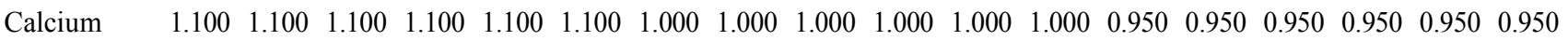

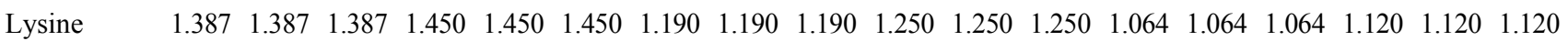

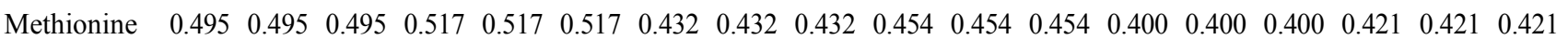

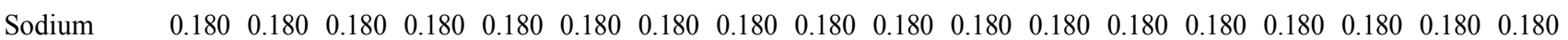
AP $\quad \begin{array}{llllllllllllllllll}0.450 & 0.450 & 0.450 & 0.450 & 0.450 & 0.450 & 0.450 & 0.450 & 0.450 & 0.450 & 0.450 & 0.450 & 0.350 & 0.350 & 0.350 & 0.350 & 0.350 & 0.350\end{array}$

${ }^{1}$ Contains per kg: vit. A, 12,000,000 IU; vit $\mathrm{D}_{3}, 5,000,000 \mathrm{IU}$; vit $\mathrm{E}, 50,000 \mathrm{IU}$; vit $\mathrm{K}_{3}, 3,000 \mathrm{mg} ;$ vit $\mathrm{B}_{1}, 2,000 \mathrm{mg}$; vit $\mathrm{B}_{2}$, $6,000 \mathrm{mg}$; vit $\mathrm{B}_{6}, 4,000 \mathrm{mg}$; vit $\mathrm{B}_{12}, 25 \mathrm{mg}$; Biotin, $200 \mathrm{mg}$; folica cid, 2,000 mg; niacin, 70,000 mg; pantothenic acid, $20,000 \mathrm{mg}$.

${ }^{2}$ Contains per kg; Cu, 20,000 mg; Co, $500 \mathrm{mg}$; Fe, 50,000 mg; I, 1,300 mg; Mn, 120,000 mg; Se, $300 \mathrm{mg} ; \mathrm{Zn}, 100,000 \mathrm{mg}$.

ments by two-way analysis of variance using the GLM procedure in SAS (9.1., Cary, NC, 2002). Duncan's new multiplerange test was performed to identify differences (Steel and Torrie, 1980). A $P$-value $<0.05$ was considered significant.

\section{RESULTS AND DISCUSSION}

The performance (BW, FI and FCR) of the broilers in experiment 1 was influenced by the interaction of $\mathrm{ME}$ and $\mathrm{CP}$ in the diet (Table 3). During the pre-starter period $(0 \sim 7$ days), performance were not influenced by the dietary $\mathrm{ME}$ and CP. Extending the grow-out period from 8 to 21 days, resulted in increased weight gain and feed intake numerically 
Table 2. General diet composition of experiment 2

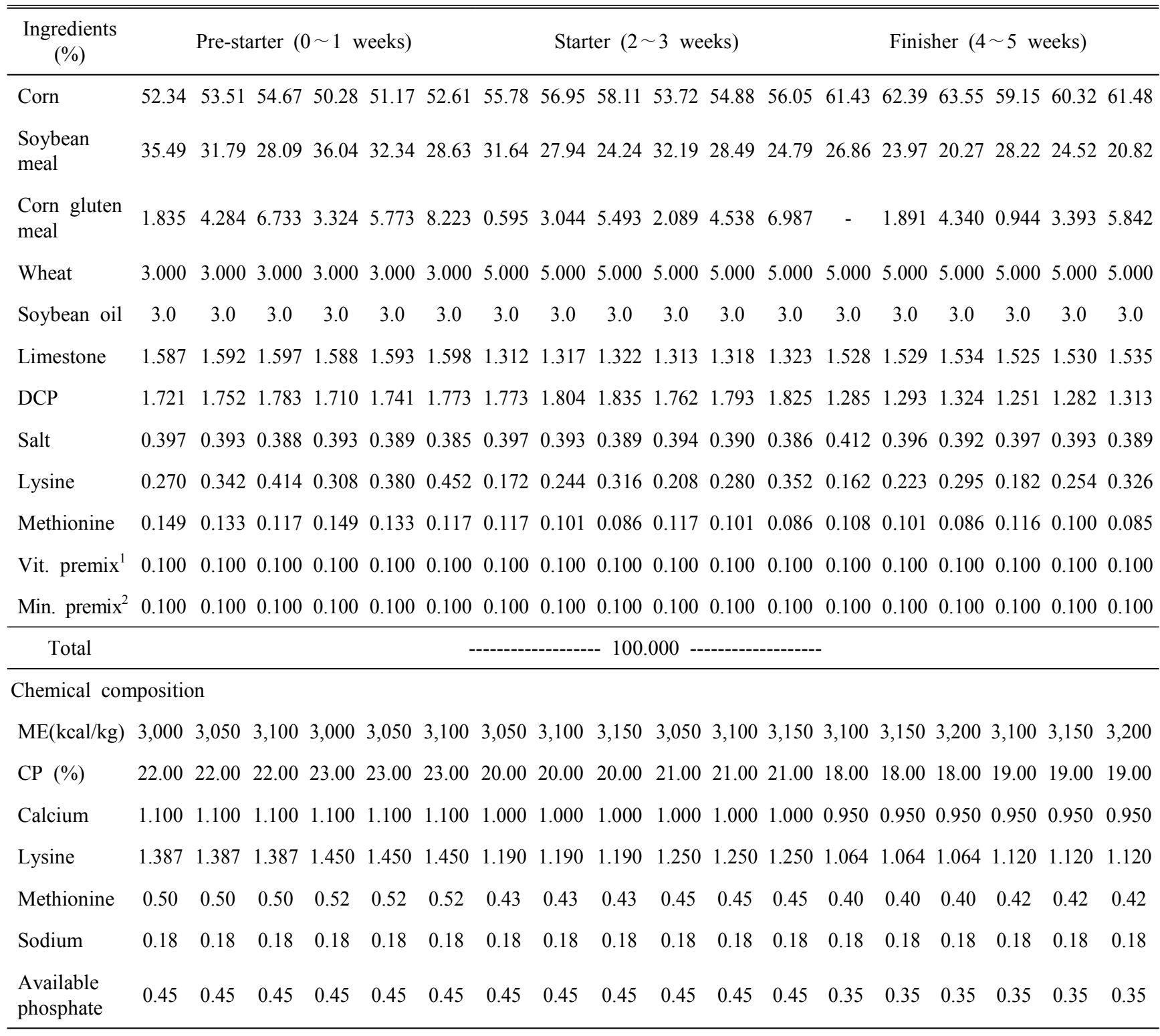

${ }^{1}$ Contains per kg: vit. A, 12,000,000 IU; vit $\mathrm{D}_{3}, 5,000,000 \mathrm{IU}$; vit $\mathrm{E}, 50,000 \mathrm{IU}$; vit $\mathrm{K}_{3}, 3,000 \mathrm{mg}$; vit $\mathrm{B}_{1}, 2,000 \mathrm{mg}$; vit $\mathrm{B}_{2}$, $6,000 \mathrm{mg}$; vit $\mathrm{B}_{6}, 4,000 \mathrm{mg}$; vit $\mathrm{B}_{12}, 25 \mathrm{mg}$; Biotin, $200 \mathrm{mg}$; folic acid, 2,000 mg; niacin, 70,000 mg; pantothenic acid, 20,000 mg.

${ }^{2}$ Contains per kg; Cu, 20,000 mg; Co, $500 \mathrm{mg}$; Fe, 50,000 mg; I, 1,300 mg; Mn, 120,000 mg; Se, $300 \mathrm{mg}$; Zn, $100,000 \mathrm{mg}$.

in chicks in $3,000 \times 20$ and $3,000 \times 21 \mathrm{kcal}$ of $\mathrm{ME} / \mathrm{kg}$ and $\mathrm{CP}$ treatments. When the growth period was extended further (22 35 days), weight gain was increased $(p<0.05)$ to the interaction of ME and CP level $(3,000 \times 18$ and 3,000 × 19) in the diet might be due to increased feed intake and thus enhanced growth performance. Although we did not measure fat deposition in the present trial, but have measured serum lipid properties (glucose, cholesterol and HDL) and which were increased numerically with the energy content in the diet. : $P>0.05$, therefore no meaning. Therefore, dietary interaction of 3,000 kcal ME and 19\% CP would enhance growth performance. In follow up studies, a small increase was observed when dietary energy was increased from 3,050 to $3,150 \mathrm{kcal} /$ $\mathrm{kg}$ on $2 \sim 3 \mathrm{wks}$ and 3,100 to 3,200 on the $4 \sim 5$ wks of age 
Table 3. Effect of different level of $\mathrm{ME}$ and $\mathrm{CP}$ on the performance of broiler chicks (Experiment 1)

\begin{tabular}{|c|c|c|c|c|c|c|c|c|}
\hline \multirow{3}{*}{ Items } & \multicolumn{6}{|c|}{ Treatments } & \multirow{3}{*}{ SEM } & \multirow{3}{*}{$\begin{array}{l}P \text { value } \\
\mathrm{ME} \times \mathrm{CP}\end{array}$} \\
\hline & \multicolumn{2}{|c|}{$3,000 \mathrm{kcal} / \mathrm{kg} \mathrm{ME}$} & \multicolumn{2}{|c|}{$3,100 \mathrm{kcal} / \mathrm{kg} \mathrm{ME}$} & \multicolumn{2}{|c|}{$3,200 \mathrm{kcal} / \mathrm{kg} \mathrm{ME}$} & & \\
\hline & $\begin{array}{c}22,20 \& \\
18 \% \mathrm{CP}\end{array}$ & $\begin{array}{c}23,21 \& \\
19 \% \mathrm{CP}\end{array}$ & $\begin{array}{c}22,20 \& \\
18 \% \mathrm{CP}\end{array}$ & $\begin{array}{c}23,21 \& \\
19 \% \mathrm{CP}\end{array}$ & $\begin{array}{c}22,20 \& \\
18 \% \mathrm{CP}\end{array}$ & $\begin{array}{c}23,21 \& \\
19 \% \mathrm{CP}\end{array}$ & & \\
\hline \multicolumn{9}{|c|}{ Weight gain (g) } \\
\hline $0 \sim 1$ wks & 124.02 & 124.14 & 117.23 & 121.24 & 126.23 & 122.39 & 1.97 & 0.7110 \\
\hline $2 \sim 3$ wks & 681.13 & 685.26 & 666.15 & 683.19 & 659.46 & 672.57 & 6.77 & 0.9429 \\
\hline $4 \sim 5$ wks & $1,032.17^{\mathrm{a}}$ & $1,021.27^{\mathrm{a}}$ & $1,013.26^{\mathrm{a}}$ & $938.16^{\mathrm{b}}$ & $950.67^{\mathrm{b}}$ & $930.61^{\mathrm{b}}$ & 12.34 & 0.0382 \\
\hline $0 \sim 5$ wks & $1,837.32^{\mathrm{a}}$ & $1,831.67^{\mathrm{a}}$ & $1,796.65^{\mathrm{ab}}$ & $1,743.59^{\mathrm{b}}$ & $1,737.36^{\mathrm{b}}$ & $1,725.57^{\mathrm{b}}$ & 13.22 & 0.0439 \\
\hline \multicolumn{9}{|c|}{ Feed intake $(\mathrm{g})$} \\
\hline $0 \sim 1$ wks & 141.24 & 132.04 & 129.18 & 139.17 & 143.21 & 133.19 & 2.52 & 0.2027 \\
\hline $2 \sim 3$ wks & $1,059.18$ & $1,068.13$ & $1,075.27$ & $1,064.38$ & $1,078.37$ & $1,045.31$ & 9.36 & 0.7040 \\
\hline $4 \sim 5 \mathrm{wks}$ & $1,919.27^{\mathrm{a}}$ & $1,979.27^{\mathrm{a}}$ & $1,908.12^{\mathrm{a}}$ & $1,766.54^{\mathrm{b}}$ & $1,831.28^{\mathrm{ab}}$ & $1,734.47^{\mathrm{b}}$ & 21.37 & 0.0382 \\
\hline $0 \sim 5$ wks & $3,119.69$ & $3,179.44$ & $3,112.57$ & $2,971.09$ & $3,053.86$ & $2,912.97$ & 27.06 & 0.1441 \\
\hline \multicolumn{9}{|c|}{ Feed conversion ratio } \\
\hline $0 \sim 1$ wks & 1.143 & 1.061 & 1.109 & 1.151 & 1.137 & 1.086 & 0.01 & 0.1228 \\
\hline $2 \sim 3$ wks & 1.556 & 1.558 & 1.618 & 1.558 & 1.637 & 1.555 & 0.01 & 0.3969 \\
\hline $4 \sim 5$ wks & 1.862 & 1.953 & 1.888 & 1.884 & 1.926 & 1.863 & 0.02 & 0.3781 \\
\hline $0 \sim 5$ wks & 1.699 & 1.741 & 1.732 & 1.705 & 1.762 & 1.688 & 0.01 & 0.2140 \\
\hline
\end{tabular}

SEM, Standard error of mean.

${ }^{1}$ First value indicates $\mathrm{CP}$ from 1 to 7 days of age; second value indicates $\mathrm{CP}$ from 8 to 21 days; third value indicates $\mathrm{CP}$ from 22 to 35 days.

a,b Means within the column followed by different letters are significantly different $(P<0.05)$.

(Table 5). It might be hypothesized that it could be happened due to the small range of ME and CP levels used in the present study. Between the feeding trials, dietary energy and $\mathrm{CP}$ had no significant effect on FCR, but feed intake was increased with decreasing dietary energy and protein dependant manner, indicating that birds can regulate their feed intake based on energy and protein consumption (Sterling et al. 2003 and Nahashon et al, 2005). In consistent, Hidalgo et al. (2004) increased dietary energy from 3,020 to $3,196 \mathrm{kcal} / \mathrm{kg}$ and reported that there were no significant differences in growth rate, feed consumption and feed conversion. Thereafter, Lesson et al. (1996 a,b) who supplied various levels of dietary energy $(2,700,2,900,3,100$ and $3,300 \mathrm{kcal} / \mathrm{kg})$ which had not significantly effect on growth rate and recommended that broilers have good ability to control its feed intake based on energy content in the diet. In another experiment, Sizemore and Sigel, (1993) reported higher growth rate, less feed consumed and improved feed conversion when chicks were fed diets formulated to $3,190 \mathrm{kcal}$ of $\mathrm{ME} / \mathrm{kg}$ compared to $2,712 \mathrm{kcal}$ of $\mathrm{ME} / \mathrm{kg}$ during the starter period ( 0 to $21 \mathrm{~d}$ ), and thus increased body weight. In contrast, Saleh et al. (2004. a,b) used various levels of $\mathrm{ME}(3,023$ to 3,383$)$ and highest growth was achieved under the $3,276 \mathrm{kcal} / \mathrm{kg}$ of ME treatment at 21 , 42 and 49 days of age $(p<0.05)$. Thus, the variation of broilers performance to the dietary energy and protein might be due to the variation of dietary $\mathrm{ME}$ and $\mathrm{CP}$ level, feed composition, age and strain of the bird.

Dietary protein and carbohydrate has an important impact 
Table 4. Effect of different level of $\mathrm{ME}$ and $\mathrm{CP}$ on the blood component and bone mineral density of broiler chicks (Experiment 1)

\begin{tabular}{|c|c|c|c|c|c|c|c|c|}
\hline \multirow{3}{*}{ Items } & \multicolumn{6}{|c|}{ Treatments } & \multirow{3}{*}{ SEM } & \multirow{3}{*}{$\begin{array}{l}P \text { value } \\
\mathrm{ME} \times \mathrm{CP}\end{array}$} \\
\hline & \multicolumn{2}{|c|}{$3,000 \mathrm{kcal} / \mathrm{kg} \mathrm{ME}$} & \multicolumn{2}{|c|}{$3,100 \mathrm{kcal} / \mathrm{kg} \mathrm{ME}$} & \multicolumn{2}{|c|}{$3,200 \mathrm{kcal} / \mathrm{kg} \mathrm{ME}$} & & \\
\hline & $\begin{array}{c}22,20 \& \\
18 \% \mathrm{CP}\end{array}$ & $\begin{array}{c}23,21 \& \\
19 \% \mathrm{CP}\end{array}$ & $\begin{array}{c}22,20 \& \\
18 \% \mathrm{CP}\end{array}$ & $\begin{array}{c}22,20 \& \\
18 \% \mathrm{CP}\end{array}$ & $\begin{array}{c}23,21 \& \\
19 \% \mathrm{CP}\end{array}$ & $\begin{array}{c}22,20 \& \\
18 \% \mathrm{CP}\end{array}$ & & \\
\hline \multicolumn{9}{|l|}{ Blood properties } \\
\hline Alb $(g / d L)$ & 1.31 & 1.18 & 1.34 & 1.25 & 1.27 & 1.14 & 0.02 & 0.8461 \\
\hline $\mathrm{TP}(\mathrm{g} / \mathrm{dL})$ & 2.66 & 2.46 & 2.66 & 2.60 & 2.64 & 2.43 & 0.03 & 0.5713 \\
\hline Glu (mg/dL) & 169.81 & 157.28 & 166.14 & 167.88 & 173.25 & 174.23 & 3.44 & 0.4828 \\
\hline TG (mg/dL) & 41.60 & 43.80 & 44.40 & 43.60 & 45.80 & 44.70 & 1.48 & 0.7798 \\
\hline $\mathrm{CHOL}(\mathrm{mg} / \mathrm{dL})$ & 121.80 & 116.00 & 131.90 & 129.90 & 137.50 & 126.60 & 2.63 & 0.7796 \\
\hline $\mathrm{HDL}(\mathrm{mg} / \mathrm{dL})$ & 92.90 & 90.50 & 94.30 & 99.30 & 105.20 & 91.50 & 2.16 & 0.2104 \\
\hline $\operatorname{AST}(\mathrm{U} / \mathrm{L})$ & 219.60 & 235.70 & 217.60 & 232.10 & 211.20 & 197.60 & 8.78 & 0.2846 \\
\hline $\operatorname{ALT}(\mathrm{U} / \mathrm{L})$ & 1.57 & 1.58 & 1.29 & 1.72 & 1.45 & 1.57 & 0.23 & 0.43 \\
\hline \multicolumn{9}{|c|}{ Tibia bone mineral density } \\
\hline $\operatorname{BMD}\left(\mathrm{mg} / \mathrm{cm}^{2}\right)$ & 165.73 & 168.02 & 166.03 & 161.61 & 170.63 & 180.61 & 0.01 & 0.3858 \\
\hline
\end{tabular}

SEM, Standard error of mean.

${ }^{1}$ First value indicates $\mathrm{CP}$ from 1 to 7 days of age; second value indicates $\mathrm{CP}$ from 8 to 21 days; third value indicates $\mathrm{CP}$ from 22 to 35 days.

a,b Means within the column followed by different letters are significantly different $(P<0.05)$.

ALB : albumin; TP : total protein; Glu : glucose; TG : triacylglyceride; CHOL : cholesterol; HDL : high density lipoprotein; AST : aspartate aminotransferase; ALT : alanine aminotransferase; BMD : bone mineral density.

on bone health (Son Sook Mee, Ye Na Chun 2004). There was no interaction between $\mathrm{ME} \times \mathrm{CP}$ on bone mineral density (BMD) of broiler chicks in this study. When dietary energy level increased from ME 3,000 to 3,200 kcal/ $\mathrm{kg}$ and $1 \% \mathrm{CP}$ level, BMD was increased numerically but the effect was non-significant (Table 4). However a further modification of dietary energy level in different growth stage (Table 6) had no additional effect on bone characteristics. So, it can be assumed that modification of energy and protein content of the diet without altering the $\mathrm{Ca}: \mathrm{P}$ ratio had no effect on bone mineral density of the broilers chicks (Kwon et al, 2009). Therefore, the present results suggested that dietary modification of ME within the range of 3,000 to 3,200 kcal $/ \mathrm{kg}$ and decreasing $1 \% \mathrm{CP}$ did not adversely affect on the bone mineral density of broiler chicks. In another experiment, Kermanshahi et al. (2011) mentioned that increased CP content in the diet significantly decreased $\mathrm{Ca}$ and $\mathrm{N}$ retention and thus decreased bone mineral content of broiler chicks.

In both experiments, as expected, serum albumin levels was increased numerically as dietary CP level was increased (Table 4 and Table 6). There was no interaction between dietary energy and protein on blood characteristics, but dietary energy increased from 3,100 to $3,200 \mathrm{kcal} / \mathrm{kg}$ in the diet had increased serum glucose level from 237.16 to $281.28 \mathrm{mg} / \mathrm{dL}$ (Table 6). So it can be assumed that increased dietary ME and $\mathrm{CP}$ increases serum glucose level via two processes (1) an enhancement of de novo lipogenesis (Rosebrough and Steele. 1985, Swennen et al., 2005) and (2) a decreased whole body fat oxidation consecutive to the rise in carbohydrate oxidation (Shah and Garg, 1996). Therefore, serum triglycerides, total cholesterol and HDL contents were tended to increase with the energy increments in the diet. In another experiment, Swennen et al. (2005) mentioned that plasma triglycerides level was increased due to energy and protein ratio in the 
Table 5. Effect of different level of $\mathrm{ME}$ and $\mathrm{CP}$ on the performance of broiler chicks (Experiment 2)

\begin{tabular}{|c|c|c|c|c|c|c|c|c|}
\hline \multirow{3}{*}{ Items } & \multicolumn{6}{|c|}{ Treatments } & \multirow{3}{*}{ SEM } & \multirow{3}{*}{$\begin{array}{l}P \text { value } \\
\mathrm{ME} \times \mathrm{CP}\end{array}$} \\
\hline & \multicolumn{2}{|c|}{$\begin{array}{c}3,000,3,050 \& \\
3,100 \mathrm{kcal} / \mathrm{kg} \mathrm{ME}\end{array}$} & \multicolumn{2}{|c|}{$\begin{array}{c}3,050,3,100 \& \\
3,150 \mathrm{kcal} / \mathrm{kg} \mathrm{ME}\end{array}$} & \multicolumn{2}{|c|}{$\begin{array}{c}3,100,3,150 \quad \& \\
3,200 \mathrm{kcal} / \mathrm{kg} \mathrm{ME}\end{array}$} & & \\
\hline & $\begin{array}{c}22,20 \& \\
18 \% \mathrm{CP}\end{array}$ & $\begin{array}{c}23,21 \& \\
19 \% \mathrm{CP}\end{array}$ & $\begin{array}{c}22,20 \& \\
18 \% \mathrm{CP}\end{array}$ & $\begin{array}{c}23,21 \& \\
19 \% \mathrm{CP}\end{array}$ & $\begin{array}{c}22,20 \& \\
18 \% \mathrm{CP}\end{array}$ & $\begin{array}{c}23,21 \& \\
19 \% \mathrm{CP}\end{array}$ & & \\
\hline \multicolumn{9}{|c|}{ Weight gain (g) } \\
\hline $0 \sim 1$ wks & 114.03 & 125.24 & 118.07 & 119.03 & 124.24 & 119.21 & 1.65 & 0.1242 \\
\hline $2 \sim 3$ wks & 722.12 & 725.17 & 684.14 & 694.12 & 681.39 & 699.16 & 6.99 & 0.8276 \\
\hline $4 \sim 5$ wks & $1,003.08$ & $1,036.06$ & $1,024.02$ & $1,030.23$ & $1,002.37$ & $1,013.34$ & 13.12 & 0.9256 \\
\hline $0 \sim 5$ wks & $1,839.21$ & $1,887.02$ & $1,826.18$ & $1,843.39$ & 1807.99 & $1,831.73$ & 15.68 & 0.7654 \\
\hline \multicolumn{9}{|c|}{ Feed intake $(\mathrm{g})$} \\
\hline $0 \sim 1$ wks & 140.28 & 146.23 & 137.36 & 140.25 & 142.04 & 133.21 & 1.53 & 0.1021 \\
\hline $2 \sim 3 \mathrm{wks}$ & $1,071.17$ & $1,065.41$ & $1,055.28$ & $1,056.34$ & $1,047.13$ & $1,048.08$ & 6.70 & 0.9717 \\
\hline $4 \sim 5$ wks & $2,059.35$ & $2,036.27$ & $1,951.12$ & $1,931.27$ & $1,957.06$ & $1,972.17$ & 23.74 & 0.7105 \\
\hline $0 \sim 5$ wks & $3,270.81$ & $3,247.92$ & $3,134.78$ & $3,116.87$ & $3,146.24$ & $3,183.46$ & 24.86 & 0.7083 \\
\hline \multicolumn{9}{|c|}{ Feed conversion efficiency } \\
\hline $0 \sim 1$ wks & 1.236 & 1.168 & 1.164 & 1.178 & 1.144 & 1.121 & 0.01 & 0.2803 \\
\hline $2 \sim 3$ wks & 1.491 & 1.471 & 1.541 & 1.523 & 1.537 & 1.498 & 0.01 & 0.7795 \\
\hline $4 \sim 5$ wks & 2.034 & 1.966 & 1.910 & 1.876 & 1.952 & 1.946 & 0.02 & 0.5650 \\
\hline $0 \sim 5$ wks & 1.778 & 1.731 & 1.719 & 1.691 & 1.740 & 1.738 & 0.01 & 0.4093 \\
\hline
\end{tabular}

SEM, Standard error of mean.

${ }^{1}$ First value indicates $\mathrm{ME}$ and $\mathrm{CP}$ from 1 to 7 days of age; second value indicates ME and CP from 8 to 21 days; third value indicates $\mathrm{ME}$ and $\mathrm{CP}$ from 22 to 35 days.

a,b Means within the column followed by different letters are significantly different $(P<0.05)$.

diet. Liver function was assessed at the end of both experiments by determining serum AST and ALT levels (Tables 4 and Table 6). In both experiments, serum AST and ALT were not influenced by the interaction of $\mathrm{ME}$ and $\mathrm{CP}$ in the diet, but birds under the 3,200 kcal/kg ME and 19\% CP dietary treatments tended to show lower activities and which indicated healthy flock. On the other hand, higher level of AST was found in Experiment 2 may be due to a particular experimental environment over time.

\section{SUMMARY}

본 실험은 육계의 서로 다른 농도의 에너지와 단백질이 생산성과 혈액성상 및 $\mathrm{BMD}$ 에 미치는 영향을 구명하기 위
하여 두 차례 실시하였다. 실험 1에서 총 480수의 Ross $\times$ Ross 병아리를 무작위로 6처리구(5반복; 16수/펜)로 나누어 배치하였다. 처리구는 사료 내 ME는 $3,000,3,100,3,200$ $\mathrm{kcal} / \mathrm{kg}$ 수준으로 하였고 $\mathrm{CP}$ 는 초기 $22,23 \%$, 전기 $20,21 \%$, 후기 $18,19 \%$ 수준으로 하였다. 실험 2 에서는 실험 1 과 유 사하게 병아리와 $\mathrm{CP}$ 를 적용하였으며, 사료 내 $\mathrm{ME}$ 수준을 변형하였다(초기 : $3,000,3,050,3,100 \mathrm{kcal} / \mathrm{kg}$, 전기 : 3,050 , $3,100,3,150 \mathrm{kcal} / \mathrm{kg}$, 후기 : $3,100,3,150,3,200 \mathrm{kcal} / \mathrm{kg}$ ). 두 실험에서 처리구당 10 수씩 혈액과 경골 샘플을 채취하였고, 혈액성상과 $\mathrm{BMD}$ 를 분석하였다. 실험 1 에서 증체량과 사료 섭취량은 $3,000 \mathrm{kcal} / \mathrm{kg}$ 의 에너지를 급여한 처리구에서 증가 되었다 $(P<0.05)$. 혈청 총 단백질과 albumin 농도는 $\mathrm{CP}$ 의 수 준이 증가함에 따라 수치적으로 증가하였다. 총 콜레스테롤 과 $\mathrm{HDL}$ 의 함유량은 에너지 함유량의 증가에 따라 수치적으 
Table 6. Effect of different level of $\mathrm{ME}$ and $\mathrm{CP}$ on the blood component and bone mineral density of broiler chicks (Experiment 2)

\begin{tabular}{|c|c|c|c|c|c|c|c|c|}
\hline \multirow{3}{*}{ Items } & \multicolumn{6}{|c|}{ Treatments } & \multirow{3}{*}{ SEM } & \multirow{3}{*}{$\begin{array}{l}P \text { value } \\
\mathrm{ME} \times \mathrm{CP}\end{array}$} \\
\hline & \multicolumn{2}{|c|}{$\begin{array}{c}3,000,3,050 \& \\
3,100 \mathrm{kcal} / \mathrm{kg} \mathrm{ME}\end{array}$} & \multicolumn{2}{|c|}{$\begin{array}{c}3,050,3,100 \& \\
3,150 \mathrm{kcal} / \mathrm{kg} \mathrm{ME}\end{array}$} & \multicolumn{2}{|c|}{$\begin{array}{c}3,100,3,150 \& \\
3,200 \mathrm{kcal} / \mathrm{kg} \mathrm{ME}\end{array}$} & & \\
\hline & $\begin{array}{c}22,20 \& \\
18 \% \mathrm{CP}\end{array}$ & $\begin{array}{c}23,21 \& \\
19 \% \mathrm{CP}\end{array}$ & $\begin{array}{c}22,20 \& \\
18 \% \mathrm{CP}\end{array}$ & $\begin{array}{c}23,21 \& \\
19 \% \mathrm{CP}\end{array}$ & $\begin{array}{c}22,20 \& \\
18 \% \mathrm{CP}\end{array}$ & $\begin{array}{c}23,21 \& \\
19 \% \mathrm{CP}\end{array}$ & & \\
\hline \multicolumn{9}{|l|}{ Blood properties } \\
\hline Alb (g/dL) & 1.33 & 1.33 & 1.29 & 1.32 & 1.28 & 1.37 & 0.02 & 0.3917 \\
\hline $\mathrm{TP}(\mathrm{g} / \mathrm{dL})$ & 2.73 & 2.82 & 2.82 & 2.90 & 2.72 & 2.83 & 0.04 & 1.4707 \\
\hline Glu (mg/dL) & $198.77^{\mathrm{c}}$ & $237.16^{\mathrm{bc}}$ & $228.60^{c}$ & $226.08^{c}$ & $281.28^{\mathrm{a}}$ & $268.56^{\mathrm{ab}}$ & 6.47 & 0.0026 \\
\hline $\mathrm{TG}(\mathrm{mg} / \mathrm{dL})$ & 79.90 & 88.80 & 89.40 & 86.60 & 90.00 & 91.30 & 2.27 & 0.5815 \\
\hline CHOL $(\mathrm{mg} / \mathrm{dL})$ & 117.00 & 127.50 & 137.40 & 131.00 & 134.50 & 140.20 & 2.34 & 0.2850 \\
\hline $\mathrm{HDL}(\mathrm{mg} / \mathrm{dL})$ & 87.20 & 91.00 & 100.30 & 90.60 & 99.30 & 103.70 & 2.11 & 0.2904 \\
\hline $\operatorname{AST}(\mathrm{U} / \mathrm{L})$ & 253.40 & 264.60 & 253.40 & 249.10 & 255.60 & 296.60 & 4.76 & 0.2417 \\
\hline $\operatorname{ALT}(\mathrm{U} / \mathrm{L})$ & 1.66 & 1.87 & 1.82 & 1.67 & 1.55 & 1.1 .72 & 0.25 & 0.578 \\
\hline \multicolumn{9}{|c|}{ Tibia bone mineral density } \\
\hline $\operatorname{BMD}\left(\mathrm{mg} / \mathrm{cm}^{2}\right)$ & 180.12 & 169.37 & 171.48 & 160.46 & 174.36 & 165.23 & 1.98 & 0.9758 \\
\hline
\end{tabular}

SEM, Standard error of mean.

${ }^{a} \sim \mathrm{c}$ Values in a row with no common superscripts differ significantly $(p<0.05)$.

ALB : albumin; TP : totalprotein; Glu : glucose; TG : triacylglyceride; CHOL : cholesterol; HDL : highdensitylipoprotein; AST : aspartateaminotransferase; ALT : alanineaminotransferase; BMD : bonemineraldensity.

로 증가하였다. 실험 2에서 증체량은 일관되게 에너지와 단 백질 수준의 증가에 따라 수치적으로 증가하였다(초기 3,000 $\times 23$, 전기 $3,050 \times 21$, 후기 $3,100 \mathrm{kcal} / \mathrm{kg} \mathrm{ME}$ 와 $19 \% \mathrm{CP})$. 혈청 glucose 수준은 에너지 수준이 높아짐에 따라 증가됐다 $(P<0.05)$. 따라서 혈청 총 단백질, albumin, triglycerides, 총 콜레스테롤 그리고 $\mathrm{HDL}$ 함유량은 사료 에너지 증가에 따라 증가하는 경향이 있다.

(색인 : 에너지, 단백질, 생산성, 혈액 육계)

\section{LITERATURE CITED}

Bartov I, Bornstein S, Lipstein B 1974 Effect of calorie to protein ratio on the degree of fatness in broilers fed on practical diets. Br Poult Sci 15: 107-117.

Griffiths L, Leeson S, Summers JD 1977 Fat deposition in broilers: Effect of dietary energy to protein balance and early life caloric restriction on productive performance and abdominal fat pad size. Poult Sci 56: 638-646.
Hidalgo MA, Dozier III WA, Davis AJ, Gordon RW 2004 Live performance and meat yield responses to progressive concentrations of dietary energy at a constant metabolizable energy-to-crude protein ratio. J Appl Poult Res 13: 319-327.

Jang IS, Kang SY, Ko YH, Moon YS, Sohn SH 2009 Effect of qualitative and quantitative feed restriction on growth performance and immune function in broiler chickens. Asian-Aust J Anim Sci 22: 388-395.

Kermanshahi H, Ziaei N, Pilevar M 2011 Effect of dietary crude protein fluctuation on performance, blood parameters and nutrients retention in broiler chicken during starter period. Global Vet 6: 162-167.

Kwon S, Lee B-K, Kim, H-S 2009 Relation between nutritional factors and bone status by broadband ultrasound attenuation among college students. J Korean Sco. Food Sci Nutr 38(11): 1551-1558.

Leeson S, Caston L, Summers JD 1996a Broiler response to 
diet energy. Poult Sci 75: 529-535.

Leeson S, Caston L, Summers JD 1996b Broiler response to energy or energy and protein dilution in the finisher diet. Poult Sci 75: 522-528.

Nahashon SN, Adefope N, Amenyenu A, Wright D 2005 Effects of dietary metabolizaable and crude protein concentrations growth performance and carcasss characteristics of French guinea broilers. Poultry Sci 84: 337-344.

NRC 1994 Nutrient Requirements of Poultry. 9th rev. ed. Natl. Acad. Sci., Washington, DC.

Pesti GM 2009 Impact of dietary amino acid and crude protein levels in broiler feed on biological performance. $\mathrm{J}$ Appl Poult Res 18: 478-486.

Rosebrough RW, Steele NC, 1985 Energy and protein relationships in the broiler. 1. Effect of protein levels and feeding regimens on growth, body composition and in vitro lipogenesis of broiler chicks. Poult Sci 64(1): 119-26.

Saleh EA, Watkins SE, Waldroup AL, Waldroup PW 2004a Consideration for dietary nutrient and energy feeding programs for growing large male broilers chickens for further processing. Int J Poult Sci 3: 1-10.

Saleh EA, Watkins SE, Waldroup AL, Waldroup PW 2004b Effects of dietary nutrient density on performance and carcass quality of male broilers grown for further processing. Int J Poult Sci 3: 1-10.

SAS Institute 2002 Guide for Personal Computers, Version
9.1. SAS Institute, Cary, NC, USA.

Steel RGD, Torrie JH 1980 Analysis of Covariance. Principles and Procedures of Statistics: A Biometrical Approach. McGraw-Hill, New York. pp. 401-437.

Shah M, Garg A 1996 High fat and high-carbohydrate diets and energy balance. Diabetes Care 19: 1142-52.

Sizemore FG, Siegel HS 1993 Growth, feed conversion and carcass composition in females of four broiler crosses fed starter diets with different energy levels and energy to protein ratios. Poult Sci 72: 2216-2228.

Son Sook Mee, Ye Na Chun 2004 Association between bone mineral density and bone nutrition indicators in elderly residing in low income area of the city. J Korean Soc. Food Sci Nutr 33(1): 107-113.

Sterling KG, Pesti GM, Bakalli RI 2003 Performance of broiler chicks fed various levels dietary lysine crude protein. Poultry Sci 82: 1939-1947.

Swennen Q, Janssens GPJ, Millet S, Vansant G, Decuypere E, Buyse J 2005 Effects of substitution between fat and protein on feed intake and its regulatory mechanisms in broiler chickens: Endocrine functioning and intermediary metabolism. Poult Sci 84: 1051-1057.

Tumova E, Teimouri A, 2010. Fat deposition in the broiler chicken: A review. Scientia Agriculturae Bohemica 41: 121-128.

(접수: 2013. 11. 14 수정: 2013. 12. 5, 채택: 2013. 12. 16) 